\title{
Pragmatic Association of the Two Evaluation Concepts of Operational Observation and Mathematical Modeling
}

\begin{abstract}
By Adel Razek
Recent developments in several theoretical and industrial concepts are closely associated to the relation of operational observation to mathematical modeling. The present work investigates first the interdependence of these two evaluation notions. An assessment of these notions is performed involving different analyses based on philosophical aspects as phenomenology and structural research. These analyses are also supported by illustrations from physics and quantum science. These analyses examine the autonomy limits of each of both concepts and their interdependence. The associate resulting from such interdependence is therefore studied. This involves different aspects characterizing such associate (couple) as its managing in time and its rulings. The immersion of the couple "observation-theory" is subsequently considered through the exploration of different representing cases showing the nature of the interdependence in this couple. The corroborating interdependence is illuminated in the case of coupled amended models. The matching interdependence is illustrated in the cases of the industrial digital twins concept and Bayesian brain theory in neuroscience. Finally, the imitating interdependence is pointed out in quantum and neuromorphic computing technologies. The conclusion of the paper underlines that mathematical modeling needs operational observation simply to be credible and that the second needs the first for deeper research. Additionally, the interdependence of this associate is valuable to the ideas of several research and industrial innovative concepts.
\end{abstract}

Keywords: observation-modeling associate, phenomenology, structural research, Bayesian concepts, quantum science, matching, imitating, corroborating.

\section{Introduction}

Topical occurrences in numerous academic and industrial conceptions are intimately linked to the associate observation modeling. These two evaluation concepts could be employed separately or in a complementary associate.

We can carry on the analysis of a real object and its behavior according to real environmental events by operational observation or theoretical modeling. Depending on the concerned area of investigation, researchers prefer often one of these two concepts to evaluate the behavior of the subject under discussion. Observation is traditionally employed in human and social sciences e.g. anthropology, psychology, epidemiology... It is also partially used in earth science, applied physics and chemistry... On the other hand, mathematical

*Emeritus Research Director, C.N.R.S. \& Honorary Professor, CentraleSupelec, GeePs, University of Paris-Saclay and Sorbonne University, France. 
modeling is often employed generally in "hard" sciences as general physics, chemistry... These two concepts are not in fact, independent or auto sufficient. The validity of each results from the benefit of the other. This insures deeper and/ or credible research outcomes.

A real observable object and its behavior according to real observable environmental events could be explored by operational observation. Simple tools or instruments that are more sophisticated could perform such observation; this depends on the complexity of the observation task. When the object has an inherent nature and behaving under a simple environmental event, we are tackling a simple observation task. In the contrary, when the object nature inclines to interact with the environment in an interdependence frame, the task will be more complicated. This complexity will be particularly stronger in the case of interaction of the object with multiple events that are interdependent. The last situation, where the nature of the object as well as the different environmental events, are interacting interdependently corresponds to numerous societal realistic application domains e.g. health, mobility, energy.... In such case, an observation result (point) will be valid for a given specified operational condition. The different observation points (data) could be treated by algorithmic technics to perform a more universal and complete analysis. Thus, even observation could be autosufficient; it still needs mathematical modeling treatments for deeper investigations.

In the case where the analyzed behavior could be governed by known and founded verified theories, another evaluation issue for this problem may be the mathematical modeling. Here we mean by modeling, using mathematical formulation based on a theory expressed under the form of mathematical expression. In general, one can consider a theory only established after validation by observation. Moreover, such a theory remains valid until disagreement with observation. Therefore, the theory-observation couple is necessarily always associated.

Founding coherent and agreeable theories habitually requires postulations that squeeze and idealize the actual context of the investigation. When the situation studied is very close to such an unrealistic context, the problem of modeling analysis could be self-sufficient. In realistic situations, to amend for idealizing approximation errors, we have to account beside the principle-studied idealized phenomenon for more phenomena concerned by the committed approximations. Thus, we will have a multiple phenomenon (events) system to model in order to approach a realistic solution.

As in the case of observation, the complexity of the task of modeling will depend on how interacts the object with its environment. Each interaction of the object with an environmental event will be considered through a consequent theoretical treatment. Therefore, the complete analysis of the behavior of the studied object could be only performed through all the theories corresponding to the different concerned events (the principle one and those corresponding to the committed approximations). Moreover, if the natures of the studied object and the environmental events are interdependent, the modeling task will go through a coupled analysis of different theories (mathematical expressions), see e.g. (Razek 2020a). Such coupling may be performed sequentially in case of linear behaviors; 
see for example in the case of electromagnetic systems (EMS), (Sekkak et al. 1994, Piriou and Razek 1990). In the general case where the events self-behaviors and/or their interdependent behaviors are non-linear, we need a simultaneous coupling; see for example again in EMS (Ren and Razek 1994).

We can have an interrogation on the self-sufficiency of each of these two issues of analysis (concepts of observation and modeling) as well as on their complementarity (Razek 2020b). The nature and field of investigation is closely related to this questioning. Observation or modeling can be self-sufficient in areas of investigation that we often consider idealized or of simple nature. In the general case of real societal landscapes, the situation is more realistic and complex. In such case we need to use the two issues of analysis in a framework of complementarity. Such association is often necessary to obtain credible and deeper investigations. Thus, the validation by observation in the case of modeling is needed for creditability (see e.g. Merleau-Ponty 1960). In addition, the extension of the research limits by modeling in the case of observation is required for deepness (see e.g. Lévi-Strauss 1958).

In the present work, an assessment of the concepts of observation and modeling is performed. This involves different analyses based on philosophical aspects as phenomenology and structural research as well as illustrations from physics and quantum science. After this assessment, we will analyze the modelobservation associate in general. This involves different aspects of the couple "theory-observation" concerns as the theory-observation managing, the rulings on theory-observation, the theory-observation couple immersion. Different illustrating cases are analyzed including amended models (coupled phenomena), digital twins concept, and Bayesian brain theory as well as quantum and neuromorphic technologies (computing).

\section{Assessment of the Concepts: Observation and Modeling}

In this part of the paper, we will examine the limits of the self-sufficiency of both concepts.

\section{Theoretical Modeling Isolated from Observation}

Examine the bounds of the autonomy of the theoretical survey, counting for the research areas, which generally require theoretical modeling, for example physical, chemical, etc. In these cases, such modeling uses mathematical formulation based on a theory expressed under the form of mathematical expression. In such situations, founding coherent and agreeable theories habitually obligates postulations that squeeze and idealize the actual context of the investigation. When the situation studied is very close to such an unrealistic context, the problem of modeling analysis could be self-sufficient. On the contrary, in most societal survey situations, this type of analysis will not be sufficient. Furthermore, for problems where the theoretical behavior (mathematical expression) is not well known or unproven, it is manifest that the 
use of isolated mathematical modeling would be hopeless and speculative; this is the case of e.g. inductive prediction (see Popper 1934).

\section{Merleau-Ponty, Phenomenology and Science}

A typical example of cases of non-self-sufficiently of modeling analysis is illustrated in several works of Maurice Merleau-Ponty (1908-1961), French philosopher and intellectual. He analyzed and commented on modeling and observation in different publications; (see for example, Merleau-Ponty 1960). Merleau-Ponty was the main academic supporter of existentialism and phenomenology. Recognized for his creative and meaningful work on incarnation, perception and ontology, he has also made important contributions to several other fields including art, history, language, and nature.

Merleau-Ponty acted a vital role in the spread of phenomenology that was initially founded by the German philosopher, Edmund Husserl (1859-1938). Phenomenology can be coarsely expressed as the maintained effort to illustrate experiences (and their own things) without theoretical inferences. Husserl advocated that only by overhanging the "natural approach", philosophy could become a distinctive and rigorous science, and he asserted that phenomenology is a science of consciousness instead of empirical things. He is one of the furthermost prominent philosophers with key adapt phenomenologists, in addition to MerleauPonty, like Heidegger, Sartre, Gadamer, Levinas, and Derrida.

The Key impacts on Merleau-Ponty philosophical thoughts include besides Edmund Husserl, Henri Bergson, Max Scheler, Martin Heidegger and Jean-Paul Sartre. In addition to this list, in fields apart of philosophy, Kurt Goldstein in neurology, Wolfgang Köhler and Kurt Koffka in Gestalt theory, as well as Marcel Proust, Paul Claudel and Paul Valéry in literary. Moreover, he inspired the poststructuralist of French thinkers after him; especially Michel Foucault, Gilles Deleuze and Jacques Derrida.

Phenomenology, in accordance with Merleau-Ponty, sets away all scientific or naturalistic explanations of phenomena in order to describe faithfully the prescientific experience that such explanations take for established. Similarly, since the world exists prior to pensive analysis or judgment, phenomenology avoids reconstructing actual experience in terms of its conditions of possibility or the activity of perception. The phenomenological reduction, on his interpretation, is not an idealistic method but an existential one, namely, the reflective effort to disclose our pre-reflective engagement with the world.

The phenomenology of Perception according to Merleau-Ponty, considers the privileging of description over scientific explanation. It stands as a philosophy that places essences back into existence and does not think that human beings and the world are comprehensible. The Phenomenology is not in opposition to the sciences that are concerned with the same phenomena, even if these disciplines approach the subject from a different perspective. Merleau-Ponty considers that science improperly forces phenomenological or perceptual classes into objective classifications. The phenomenology of perception must progress from the world categories to those under which we perceive it. Science (and particularly empiricism), incorrectly infers the world of (persons, objects, actions...), from the 
reality to an associated artifact.

Built on these thoughts, Merleau-Ponty noted in several masterpieces on the limits of theoretical investigation and more generally on the science and theoretical researchers using theories set apart from reality. The ultimate one of these oeuvres was "The eye and the spirit, Paris 1960" mentioned before. He noticed, as a Professor at "Collège de France" and at the Lyon University, concerning isolateduse of theory, that science handles things, abandons inhabiting inside and considers the world as an item of knowledge "disconnected" from the subsisting subject. In addition, he complemented with respect to the models, that they are closely contingent to their authors, that there is correlation between the model and the mind, and that the scientists perceive the world with a spirit linked to the model discounting the reality observation.

The Merleau-Ponty thoughts suggest, as mentioned before, the presence of a correspondence between the model, its author and the minds of its users. Almost fifty years later, and thanks to research in modern neuroscience, the theory of mirror neurons has confirmed this proposition. This research first showed for animals, then for humans, thanks to functional MRI brain mapping, that the zones of activated neuronal connections are similar, involving an observable and one or more observers.

\section{Parity Violation in Weak Interactions (in Physical Science)}

After the illustration of the weakness of isolated theoretical modeling from the philosophical point of view, we will consider an additional illustration relative to an example of important discovery in the 1950s in the field of subatomic elementary particles.

It is well known that symmetries play an important role in physics. In addition, parity was naturally assumed to be conserved in the four fundamental interactions: electromagnetism, gravity (long-range forces) \& strong and weak interactions (subatomic). This was believed until Lee and Yang (Nobel 1957) suggested theoretically in 1955 parity violation in weak interactions. A fast experimental confirmation (by $\mathrm{Wu}$ ) has been performed immediately after such theoretical proposal. This experimental validation was crucial for the establishment of this fact and instituted it simply credible. It may be noted that this contribution originated numerous explorations in elementary particles (subatomic) concerns.

\section{Sound Tongue of Feynman}

One more picture of the weakness of isolated modeling concerns a famous statement of Richard Phillips Feynman (1918-1988), father of Quantum electrodynamics (Nobel 1965) and one of the firsts proposing Quantum computing. The statement is: "No matter how beautiful your theory is, No matter how smart you are, No matter if you're famous ...If your theory is not in tune with experience, It is wrong. That's all".

\section{Weakness of Isolated Theoretical Modeling}

The last sections relative to the position of theoretical isolated investigations, supported largely by the philosophical point of view of Merleau-Ponty and 
reinforced by subatomic and Quantum physics research, illustrated the limits of such investigations. As a conclusion for the limits of theoretical investigation selfsufficiency, we can say that theoretical modeling used isolated could nosedive and needs observation, simply to be credible or to get equitable result.

\section{Observation Isolated from Theoretical Modeling}

Consider domains of investigations that generally call for observation. These concern traditionally human and social sciences. In such case, we need a relatively high number of observed data for deep investigations in a given situation. Despite this richness of data, the observation alone rapidly appeared limiting the extension of the field of research. Putting such observational studies in a theoretical context opens the field of exploration of the concerned situation. In addition, these theoretical "tools" make it possible to generalize a more in-depth research involving different studied situations amalgamated in the same model (theory). Consequently, these solitary observations may support into a more general structured concept illuminating real widespread phenomena and permits the enlargement from a single situation to more universal setups.

\section{Claude Lévi-Strauss and Structural Research}

A typical domain among those using observation is Anthropology. This field has used principally observation since its creation. Claude Lévi-Strauss (19082009) has illustrated the use of a theoretical structured concept (mainly mathematical) based on observational data, in "Structural Anthropology", Paris 1958, (see Lévi-Strauss 1958). Structural anthropology is one of the founding branches of the structuralist paradigm in anthropology, developed from the 1940s by Claude Lévi-Strauss, of which it constitutes the major work. This concept is indirect consequences of modern mathematics: logic, sets, groups and topology.

Important in France, the term social anthropology (general science of society), it aims to apply to this discipline the concept in the emerging age of structuralism. The object is to explain the diversity of facts by blending a limited number of logical possibilities linked to the architecture of the human brain; thus, breaking with the dominant currents of this epoch in ethno-anthropology: evolutionism, culturalism, and functionalism. It uses the general principles of understanding a society as a complex system provided with invariable independent (structural) properties arising from the relations between the individuals that compose it.

The expression structural analysis in anthropology has often been used as synonym of structural anthropology by Lévi-Strauss himself, who fixed them as the titles of several of his articles and works. Today, these different terms remain attached to its name and continue to designate its general work and its methodology. In order to assign generally the use of the structural paradigm in anthropology, among other authors, the term usually used is that of structuralism in anthropology.

Structural anthropology, resulting from various intellectual affiliations of universal orientation, will gradually develop an emerging scientific paradigm very close to the general current and to the cognitivism that were formed at the same 
time. This takes into account the dialectical structure, the relationships within and between systems, and its ambition to describe human "mental insertions".

Even that Lévi-Strauss first used the term structuralism; he firmly and very early on dissociated himself, in the 1950s, from the resumption of his methods of analysis by a vast transdisciplinary intellectual movement of formalist inspiration. In the 1970s, Lévi-Strauss abandoned all reference to the notion of structure, to mark the major paradigm difference that separated it from the politicized and ultraformalist evolution of so-called generalized structuralism.

The example of Structural Anthropology of Lévi-Strauss illustrates the fact that structural researches in the social sciences are indirect consequences of modern mathematics. This shows clearly that observation alone, even may be auto sufficient in certain situations, needs generally the modeling issue complementarity.

\section{Michael Faraday and Electromagnetic Induction}

In physical science, we can consider the example concerning the work of Michael Faraday (1791-1867) in the field of electromagnetic induction. Faraday was an excellent observer who conveyed his ideas from his experimental work in a very humble manner. His math skills, nevertheless, are limited to the unsophisticated algebra. The contributions of Faraday and others to put them in a mathematical framework and condense them into a set of equations motivated James Clerk Maxwell (1831-1879). These "Maxwell's equations" are the basis of all modern theories of electromagnetic phenomena and are the source of much scientific research in this area. This complementarity (modeling aiding observation) exists in many other fields of investigation in all disciplines of science, for example, demography, sociology, neurosciences, and physical sciences.

\section{Weakness of Isolated Observation}

The last sections relative to the position of observational isolated investigations supported largely by the structuralism of Lévi-Strauss and reinforced by the extension of Faraday work by Maxwell in electromagnetics research, illustrated the limits of such investigations. As a conclusion for the limits of observational investigation self-sufficiency, we can say that operational observation used isolated could frustrate investigations and needs theoretical modeling, obviously to permit deeper research.

\section{Theory-Observation Couple Concerns}

\section{Theory-Observation Managing and Rulings}

As discussed before, the couple theory-observation is always strongly associated. In this section, we will examine how these two associated concepts comes one to the other in time.

\section{The Usual Situation}

In the standard case the theory comes first and it is validated (or not) later by 
experience. Most of important discoveries are in this category.

We can take, for the occurrence of theory validation, as example the case of the theory of the superposition states launched (proposed) by E Schrödinger in 1930 (Nobel 1933) and validated by S Haroche \& D Wineland in 1996 (Nobel 2012). Only after such validation, this theory became established (founded) until a possible upcoming invalidation.

Concerning the theory invalidation occurrence, we can consider the example of the Hall Effect proposed by E Hall in 1879. This proposition obtained from observation (experience) has invalidated a part of the Treatise on Electricity and Magnetism proposed by JC Maxwell in 1873, concerning the relation between the force and the current in a conductor.

\section{Experience-First Situation}

Apart from the last standard case of the interiority of theory regarding experience, we can meet the situation of finding experience first and then establishing the corresponding theory afterward. In general, we meet such case in a "serendipity situation": one discover something while searching for another. A typical example is the discovery of the superconductivity by Kamerlingh Onnes (1853-1926), Nobel 1913. In this case, he was investigating problems relative to effects of low temperature on electronics without imagining the phenomenon he has observed.

\section{Simultaneous-Works Situation}

A third situation concerns simultaneous separated theoretical and experimental contributions. An emblematical case concerns the Duality Particle-Wave in quantum physics. Louis Victor de Broglie (1892-1987) Nobel 1929 has proposed the theory, and the accidental experimental validation has been simultaneously revealed by Clinton joseph Davisson (1881-1958) Nobel 1937. These two researchers ignored each the other and a third researcher (Max Born) in a conference talk has given the broadcasting of both contributions.

\section{$\underline{\text { Same-Work Situation }}$}

Another circumstance concerns the case where both theoretical and experimental works are developed in the same research. This is the other emblematic case of the discovery of Graphene and the generalizing to the Dirac cone material theory in quantum physics, work of Andre Geim \& Konstantin Novoselov Nobel 2010.

\section{Conclusions}

In all the last situations, it is worthy notable that both observation and theory encounters each, important evolutions due to the other. Moreover, "Serendipity" plays an important role in each of the two investigations concepts.

From this part, we can summarize the rulings on Theory-Observation couple as follows. In general, one can consider a theory only established after validation by observation. Moreover, such a theory remains valid until disagreement with observation. Therefore, the theory-observation couple is necessarily always 
associated.

\section{Theory-Observation Couple Immersion}

In this section, we will discuss some typical circumstances where the couple theory-observation is playing the essential role in the functioning of the phenomenon, the organ, the device...

\section{Amended Coupled Models}

One interest of theory-observation couple is the amended models accounting for more theory to fit reality. As we know, a societal observable is just, and its theoretical model is necessary. When the model does not match the societal observable, the first has to be adapted to fit better the reality. In such case, we need revised models by accounting for more theory in the model. The observed (societal) behavior is dominated by different sciences and/or involved phenomena. Therefore, a compound model will substitute the simple model. If the mathematical models concerned are particularly interdependent, only a coupled solution of these models can give an acceptable answer. The models interdependence is strongly guided by their time evolutions proximity and their self and mutual stuff behavior laws density. The degree of interdependence corresponds directly to the degree of coupling required. The limits of such degree could be defined as weak and strong. In the weak one, we consider the models individually in an iterative process linking their stuff behavior laws. In the strong, we consider a simultaneous solution of models enclosing their behavior laws. For a given degree of coupling, we need to opt for an appropriate mathematical formulation, suitable space and time scales and a proper resolution method, see e.g. (Carpes et al. 2000, Ouchetto et al. 2007, Rapetti et al. 2002).

In all cases, these amended models have to be validated by experience by means of real laboratory device, on which precise measurements can be accomplished. A typical example for such case concerns a coupled electromagnetic-mechanical model for thin conductive plate deflection analysis (Ren and Razek 1990, Razek 2020a). In this work the coupled model has been validated in two ways by analytical solution and by the TEAM (Testing Electromagnetic Analysis Methods) workshop. In the first case, an analytical solution has been developed for simple geometries and linear materials. The model has been validated using such analytical solution just for verification. The model has then been validated for the case of the TEAM Problem 12: Cantilevered beam in crossed field. It corresponds to a coupled problem with moving conductor. The device is constituted of a clamped beam, which is placed in a uniform constant magnetic field in the beam direction and a variable one exponentially decaying with time perpendicular to beam. In this case, the induced current in the beam due to the variable field will interact with the uniform field and creates a force perpinducal to the beam that will cause its movement. This TEAM problem has precisely defined dimensions, constitutive laws of materials, excitations... It is backed by a real laboratory device, on which measurements can be accomplished. 


\section{Prediction versus Observation}

Prediction is successfully operated in many consistent situations where it is associated to observation to produce accurate useful outcome. The most popular theory of the Bayesian brain in neuroscience and the concept of Digital Twins are typical illustrations of such a case, as we will see in the next sections. Furthermore, the prediction (or estimation) is often employed in the control of industrial systems and permits associated to observation (estimator-observer control), to obtain precise and quick performance, see e.g. (El Moucary et al. 2002, Baier and Meier 2020).

In contrast, the association prediction-observation has to be used with constraint in some particular situations. As mentioned before mathematical modeling can be only performed with established and validated theories. In case of problems where the theoretical behavior (mathematical expression) is not well known, or phenomenon behavior varying arbitrary, it is manifest that the use of isolated mathematical modeling would be hopeless and speculative. In such case, the use of inductive prediction: this is where one might draw a conclusion about the future using information from the past, would lead to erroneous result (Popper 1934). A typical example of such an invalid prediction could be encountered in the modeling of complex contagion behaviors such as infections by an unknown mutant virus, (Beauchemin and Handel 2011). In such a case one needs to deduce an approximated mathematical behavior (the real one is unknown) of the epidemic infection (Enserink and Kupferschmidt 2020) by the mutant unknown virus from the case of some infected subjects to apply it for future infections of others. Such methodology may help in the orientation of the observation research protocols but not in obtaining definite results. The observation will be the principle issue to solve such type of problems assisted by prediction.

These examples of the use of prediction illustrate that its use, together with observation, leads to a good end, otherwise it fails.

\section{Digital Twins Concept}

Recently the theory-observation couple has been used in a frame of the Bayesian couple of the Digital Twins concept. Digital Twins (DT) are using integrated multi-phenomena, multiscale, probabilistic approach. The concept is based on a matched real object (process, product or service) with a real-time digital (virtual) item that is a replica of the real object, and their connections (sensors, history...). The DT incorporating Internet of Thigns (IoT), Artificial Intelligence (AI), Machine Learning (ML), Software Analytics (SA)...to create living digital simulation models that update and change as their physical counterparts change. DT-assisted systems permits: Data analysis, System monitoring, Diagnostics, Problem anticipation, Future planning...

\section{Bayesian Brain}

The most popular theory relative to the functioning of the brain in neuroscience is that of the Bayesian brain. It is a typical illustration of the consistent successful use of prediction (modeling) based on observation.

In this circumstance, after a cerebral sensory observation (vision, smell, 
hearing, etc.) the predictive model creates cerebral expectations of the observed phenomenon from historical learnt data; only the error (prediction versus observation) is represented in the neuronal discharges spread out to identify the nature of such an observation. It can be noted that in this case, the predictive model is managed by a highest supercomputer (Human brain: $10^{11}$ neurons, each tied to $10^{4}$ others).

\section{Quantum and Neuromorphic Technologies (Computing)}

These two theoretical modeling tools comes directly from two observed realities belong to quantum physics and neurosciences.

In the case of quantum computing, the origin of the idea appeared at the dawn of the last century. Max Planck suggested that a "perfect black body" emits and absorbs electromagnetic waves in the form of discrete energy packages called "quanta". It was the emergence of quantum mechanics. This provided researchers by exceptional tools for the interpretation the atomic world. Later, the concept of states of quantum mechanics establishes the footing of "Quantum computers", expression invented by Richard Feynman (Feynman 1982).

A classical computer performs calculations using bits; that are 0 representing off and 1 representing on. It uses transistors to process information in the form of sequences of zeros and ones called computer binary language. More transistors correspond to more processing ability. A quantum computer uses qubits through the laws of quantum mechanics relative to the states of particles. For a qubit, a particle can be in multiple states simultaneously; this phenomenon is called superposition. Another phenomenon concerns the particles states called entanglement. This denotes when two qubits in a superposition correlate with one another; meaning the state of one depends on the state of the other. Due to these phenomena, a quantum computer can achieve 0,1 , or both states at the same time for a qubit or an entanglement of qubits. Therefore, a quantum computer with $n$ qubits can simultaneously operate on all the $2 \mathrm{n}$ possibilities, while a classical computer with $n$ bits can operate on only one of those $2 n$ possibilities at a time; thus, the first giving us more processing power. Researchers agree that quantum computers are theoretically exponentially faster and much smarter in breaking codes that are supposedly impossible for classic technology to achieve (Fedorov et al. 2018, Castelvecchi 2017).

The previous discussion shows obviously the tightened relation between the observed reality and the mathematical modeling (quantum physics - quantum computing).

Concerning neuromorphic computing, as in the case quantum computing, neuromorphic computing is not new, it is a concept advanced by Carver Mead in the end of the 1980s, relating the practice of very-large-scale integration (VLSI) systems enclosing analog circuits to mimic neuro-biological constructions existing in the nervous system (Mead 1990).

Modern advances in the AI have reintroduced attention in neuromorphic computers. The increasing attractiveness of deep learning and neural networks has encouraged a competition to develop AI hardware dedicated for neural network computations. Among the trends that have arisen in the past few years is 
neuromorphic computing, which has shown potential because of its resemblances to biological and artificial neural networks (BNN and ANN). These tools are largely used in optimization, diagnostics, images, machine learning, AI...

\section{Conclusions}

In this article, we studied the interdependence of the two evaluation notions of observation and modeling. We have proposed an evaluation of these notions involving different studies based on philosophical aspects such as phenomenology and structural research. These investigates were also supported by illustrations from physics and quantum science. They examined the limits of autonomy of each of the two concepts and their interdependence.

The associate resulting from such interdependence is therefore considered. This involved different aspects characterizing such an associate such as its management over time and its rulings. The engagement of the "observationtheory" couple was then reflected through the exploration of different illustrative cases showing the nature of the interdependence in this couple.

Corroborating interdependence has been brought to light in the case of coupled models. The matching interdependence has been illustrated in the cases of the concept of industrial digital twins and Bayesian Brain theory in neuroscience. Finally, imitating interdependence has been demonstrated in quantum and neuromorphic computing technologies.

In general, we can conclude that mathematical modeling requires operational observation simply to be credible and that the second requires the first for further research. In addition, the interdependence of this couple is valuable for the ideas of several innovative research and industrial concepts.

\section{References}

Baier NU, Meier T (2020) Using model predictive control to modulate the humidity in a broiler house and effect on energy consumption. Athens Journal of Technology and Engineering 7(3): 157-184.

Beauchemin C A, Handel A (2011) A review of mathematical models of influenza infections within a host or cell culture: lessons learned and challenges ahead. BMC Public Health 11(S1-7): 1-15.

Carpes WP Jr, Pichon L, Razek A (2000) A 3D finite element method for the modelling of bounded and unbounded electromagnetic problems in the time domain. International Journal of Numerical Modelling: Electronic Networks, Devices and Fields 13(6): 527-540.

Castelvecchi, D (2017) Quantum computers ready to leap out of the lab in 2017. Nature News 541(7635): 9-10.

El Moucary C, Mendes E, and Razek A (2002) Decoupled direct control for PWM inverter-fed induction motor drives. IEEE Transactions on Industry Applications 38(5): 1307-1315.

Enserink M, Kupferschmidt K (2020) With COVID-19, modeling takes on life and death importance. Science 367(6485): 1414-1415. 
Fedorov AK, Kiktenko EO, Lvovsky AI (2018) Quantum computers put blockchain security at risk. Nature 563(7732): 465-467.

Feynman RP (1982) Simulating physics with computers. International Journal of Theoretical Physics 21(6-7):467-488.

Lévi-Strauss C (1958) Structural anthropology. Paris.

Mead C (1990) Neuromorphic electronic systems. Proceedings of the IEEE 78(10): 16291636.

Merleau-Ponty M (1960) The eye and the spirit. Paris.

Ouchetto O, Zouhdi S, Bossavit A, Griso G, Miara B, Razek A (2007) Homogenization of structured electromagnetic materials and metamaterials. Journal of Materials Processing Technology 181(1-3): 225-229.

Piriou F, Razek A (1990), Numerical simulation of a nonconventional alternator connected to a rectifier. IEEE Transactions on Energy Conversion 5 (3): 512-518.

Popper, KR (1934) The logic of scientific discovery. English Version. London: Hutchinson.

Rapetti F, Maday Y, Bouillault F, Razek A (2002) Eddy-current calculations in threedimensional moving structures. IEEE Transactions on Magnetics 38(2): 613-616.

Razek A (2020a) The elegant theory, the observed societal reality and the potentialities of coupled models (Invited). In International Symposium on Numerical Modeling towards Digital Twin in Electrical Engineering, Beijing, China, January 5-7.

Razek A (2020b) The observable, the theory, and prospective revised models for societal concerns. Athens Journal of Sciences 7(1): 1-14.

Ren Z, Razek A (1990) A coupled electromagnetic-mechanical model for thin conductive plate deflection analysis. IEEE Transactions on Magnetics 26(5): 16501652.

Ren Z, Razek A (1994) A strong coupled model for analyzing dynamic behaviors of nonlinear electromechanical systems. IEEE Transactions on Magnetics 30(5): 32523255.

Sekkak A, Pichon L, Razek A (1994) 3-D FEM magneto-thermal analysis in microwave ovens. IEEE Transactions on Magnetics 30(5): 3347-3350. 
